\title{
»Alles, was ich jetzt noch kriege, ist ein Ge- schenk.» Die Hinwendung zum Leben durch die Konfrontation mit dem Tod
}

Sandra Adami

\section{Sprechen über den Tod}

\author{
"Jeder Satz, der über den Tod gesprochen wird, zehrt von \\ der Spannung, die zwischen der Redseligkeit des lebendigen \\ Menschen und der bedrohlichen Stummheit des Toten herrscht." \\ Thomas Macho, Todesmetaphern ${ }^{1}$
}

Immer wieder gibt es Versuche, uns mit dem Ende unseres Lebens auseinanderzusetzen, den Tod und das Sterben zu antizipieren. Und gleichzeitig ist der Tod ein Tabuthema, das uns allzu oft zum Schweigen bringt. In der Palliativmedizin und in der Hospizbewegung wird versucht, dieses Schweigen zu brechen, den Tod wieder ins Leben zu integrieren. Das beginnt häufig bei der Sprache. Darf über Tod und Sterben gesprochen werden, oder wird der Tod im wahrsten Sinne des Wortes »tot-geschwiegen «? ? Der demographische Wandel sowie drängende sich daraus und aus dem medizinischen Fortschritt abgeleitete Fragen für Entscheidungen am Lebensende können Gründe dafür sein, dass die Palliativmedizin eine zunehmende Bedeutung innerhalb der Gesellschaft erhält.

Psychologisch betrachtet stellt das Sprechen über den Tod eine Herausforderung dar, sich einem Phänomen zu nähern und gleichzeitig kontrolliert davon zu distanzieren. Mittels Sprache und Geschichten ist es uns möglich, das Was und das Wie unserer Konfrontation mit der Endlichkeit zu bestimmen. Doch wann sprechen wir über die eigene Endlichkeit? Häufig wird die eigene Endlichkeit dann bedeutsam, wenn sie in unser Leben rückt: nämlich dann, wenn wir uns zum Beispiel durch Krankheit unserer Vulnerabilität bewusst werden.

\footnotetext{
1 Thomas H. Macho, Todesmetaphern. Zur Logik der Grenzerfahrung, Frankfurt a.M. 1987,8 .

2 Reimer Gronemeyer, Wie wir dem Tod wieder einen Platz in unserem Leben einräumen können, Frankfurt a.M. 2007.
} 
Die Verwobenheit von Geschichten mit unserer Identität veranlasste mich, als Psychoonkologin und Psychotherapeutin die Auseinandersetzung mit der Endlichkeit in Erzählungen von Krebspatienten $^{3} \mathrm{zu}$ erforschen. Im Folgenden möchte ich die Ergebnisse meiner Forschung dazu nutzen, einen Bogen von den Erzählungen der Krebspatienten, die mit dem Tod konfrontiert sind, zur Annäherung an die existenzielle Auseinandersetzung mit dem Leben in menschlichen (Krisen-)Situationen zu schlagen.

\section{Die Konfrontation mit der eigenen Endlich keit durch eine schwere Erkrankung und deren sprachliche Verhandlung - Ergebnisse einer empirischen Analyse ${ }^{4}$}

Im Rahmen des Projekts krankheitserfahrungen.de ${ }^{5}$ wurden mit 42 Patientinnen und Patienten mit Darmkrebs narrative Interviews mit einem strukturierten Nachfrageteil geführt. In einer Sekundäranalyse, im Speziellen einer Supraanalyse, ${ }^{6}$ untersuchte ich die Formen, wie die Endlichkeit in den Erzählungen vorkam und wie die Endlichkeit

3 Die männliche und weibliche Schreibweise alternieren im Folgenden unsystematisch. In der weiblichen Schreibweise sind die Männer ebenso gemeint wie umgekehrt die Frauen in der männlichen Schreibweise.

${ }^{4}$ Hier sei auf die Forschungsarbeit verwiesen, aus der die empirischen Ergebnisse stammen: Sandra Adami, Zwischen Annäherung und Distanzierung. Die sprachliche Verhandlung der Konfrontation mit der eigenen Endlichkeit bei der Diagnose Darmkrebs. Eine qualitative Analyse, Freiburg i.Br. 2015.

5 Das Projekt krankheitserfahrungen.de stammt ursprünglich aus Großbritannien und hat zum Ziel, Erfahrungen mit Gesundheit, Krankheit und Medizin auf einer Internetplattform kosten- und barrierefrei zur Verfügung zu stellen. Dafür werden pro Krankheitsthema narrative Interviews mit Betroffenen gefüht, nach qualitativen Methoden ausgewertet und schließlich zu Thementexten mit illustrierenden Originalzitaten in Audio-,Video oder Textform aufbereitet. Bei der angewandten Samplingstrategie handelt es sich um ein "purposeful sampling" mit dem Ziel, eine maximale Varianz der Themen, Krankheitsstadien und der demographischen Faktoren herzustellen. Siehe: www.krankheitserfahrungen.de.Vgl. hierzu: Andrew Herxheimer/Sue Ziebland, Das DIPEx-Projekt: Eine systematische Sammlung persönlicher Krankheitserfahrungen, in: Neurolog. Rehabilitation 14 (2008), 31-40; Gabriele LuciusHoene, Das Leben mit der Krankheit: Chronisch Kranke erzählen, in: Berliner Ärzte 2 (2012), 29-31; Pete Smith, Patienten als Experten im Leben mit ihrer Erkrankung, in: Ärzte Zeitung 71 (2014), 18-19.

${ }^{6}$ Irena Medjedović, Qualitative Sekundäranalyse. Zum Potenzial einer neuen Forschungsstrategie in der empirischen Sozialforschung, Dordrecht 2014; Janet Heaton, Reworking qualitative data, London 2004. 
narrativ ausgehandelt wurde. Mithilfe von Codierungen ${ }^{7}$, Kategorienbildungen ${ }^{8}$ und narrativen Analysen ${ }^{9}$ wurden die Erzählungen der Darmkrebspatienten analysiert, um deren subjektive Erlebenswelten $\mathrm{zu}$ verstehen und verschiedene sprachlich-interaktive und performatorische Verhandlungen der Endlichkeitsthematik sichtbar zu machen. Daneben wurde der Bewältigungsaspekt des Erzählens in Bezug auf die Konfrontation mit der Endlichkeit untersucht.

Es ließen sich vielfältige Topoi herausarbeiten, wie zum Beispiel Geschichten über den Tod von anderen, Geschichten über die Planung des eigenen Abschieds, die Aushandlung von medizin-statistischen Prognosen im Zusammenhang mit Unsicherheit, Geschichten über das Aufwachen aus der Narkose. Innerhalb der verschiedenen Topoi war es den Erzählerinnen möglich, sich sowohl von ihren aufkommenden Gefühlen zu distanzieren als auch sich diesen anzunähern. Die eigene Erkrankung und insbesondere die Lebensbedrohung konnten insofern in das eigene Leben integriert werden, als dass sie als Teil der eigenen (narrativen) Identität geformt werden konnten. Handlungsmächtigkeit, Beruhigung und Selbstbestimmung auf der einen Seite und Ohnmacht, Angst und Unsicherheit auf der anderen Seite prägten das Spannungsfeld der Erzählungen.

\section{Todesmutig weiterleben - Lebenssinn und der Blick in die Zukunft}

Einen zentralen Aspekt der Ergebnisse möchte ich an dieser Stelle gerne herausgreifen und diskutieren, nämlich die Form, wie einige der Erzählerinnen ihre Vorstellung der Zukunft im Hinblick auf die erlebte Lebensbedrohung narrativ gestalteten. Die folgenden Auszüge aus den Interviews zweier an Darmkrebs erkrankten Frauen sollen veranschaulichen, was damit gemeint ist, wenn wir davon sprechen, dass Menschen im Zuge einer lebensbedrohlichen Krankheit ihr Leben neu ordnen oder überdenken. ${ }^{10}$

\footnotetext{
7 Johnny Saldana, The Coding Manual for Qualitative Researchers, Los Angeles 2013.

8 Michael Quinn Patton, Qualitative Research and Evaluation Methods, London 2002.

9 Gabriele Lucius-Hoene/Arnulf Deppermann, Rekonstruktion narrativer Identität. Ein Arbeitsbuch zur Analyse narrativer Interviews, Wiesbaden 2004.

${ }^{10}$ Im Original wurden die Erzählungen nach dem gesprächsanalytischen Transkriptionssystem transkribiert, so dass die sprachlichen Besonderheiten wie Pausen, Betonungen und Interjektionen interpretiert werden konnten. Hierauf wird an dieser Stelle der Komplexität wegen verzichtet; die Originalzitate werden als dem Schrift-
} 
Auf die Frage der Interviewerin "Gibt es denn etwas, wo Sie sagen, das wünschen Sie sich noch für die Zukunft? antwortete Frau Witt, eine Frau in den 50ern, wie folgt:

»Ich habe, als ich im Krankenhaus lag, so eine Überlebensliste aufgestellt, für mich selber. Aber das ist eine ganz intime, eine ganz eigene und ein ganz private Liste. Und die habe ich für mich aufgestellt mit Dingen, die ich einfach noch erleben wollte, im wahrsten Sinne des Wortes. Und das waren einige Dinge, die mir wichtig waren. Manche Kleinigkeiten, manche Gespräche, die ich führen wollte, manche Dinge, die ich klären wollte. Einfach um so sagen zu können:Wenn ich jetzt sterben muss, willst $\mathrm{du}$ das aber noch abhandeln und erledigen. Und das habe ich gemacht. Und diese Dinge, das hatte nichts mit irgendwelchen Reisen zu tun oder mit irgendwie was, haben wahrscheinlich ganz viele, die sagen: Okay, wenn mein Leben jetzt noch ein halbes Jahr dauern würde, dann würde ich vielleicht noch mal eine Weltreise machen oder irgendwie großartige Sachen. Habe ich nicht. Das waren bei mir mehr so ideelle Sachen oder Dinge, die halt mit zwischenmenschlichen Beziehungen zu tun hatten, oder Sachen, die einfach geklärt werden mussten, oder Dinge, die mir einfach wichtig waren. Und das ist auch alles abgehakt und alles erledigt. Und auch das ist irgendwie so ein Thema, was ich mit meiner Tochter ganz oft bespreche. Ich weiß, dass ich damals, als meine Mutter gestorben ist, da war ich ja selber erst Mitte 20, unendlich gelitten habe. Und heute noch da wahnsinnig traurig drüber bin, weil das so von jetzt auf gleich kam; also innerhalb von drei Wochen Diagnose und Tod. Drei Wochen. Und das war einfach nur fürchterlich. Und die ganze Familie war traumatisiert. Und ich habe das schon ein paar Mal mit meiner Tochter so zum Thema gebracht, dass ich ihr einfach auch erklärt habe: Wenn ich jetzt sterben müsste, dass es alles gut ist. Dass ich ein unheimlich tolles Leben bis jetzt gehabt habe, ganz viele Dinge erleben durfte; ganz viele Höhen, aber auch ganze viele Tiefen durchlebt habe. Aber dass es mein Leben ist und war. Und dass es ein unheimlich tolles Leben ist. Und ich finde das gar nicht schlimm, überhaupt nicht schlimm, wenn man gehen muss. Ich finde das Allerschlimmste ist immer nur, wenn man sterben muss und ganz viele Dinge unerledigt hat.

deutschen angepasste Fließtexte wiedergegeben. Bei den Namen handelt es sich um Pseudonyme; die Interviewpartnerinnen haben der Verwendung für Forschungszwecke zugestimmt. 
Und der große Vorteil meiner Krankheit war, dass ich alles, was ich so erledigen wollte oder erleben wollte, auch erlebt habe. Und alles das, was so danach gekommen ist, ist eigentlich für mich ein riesengroßes Geschenk, weil es so nicht mehr geplant war, einfach alles, das was jetzt da ist, so zusätzliche Zeit ist. Und ich genieße mein Leben unglaublich.»

Frau Steinbach, eine andere Patientin mit Darmkrebs in den 60ern, die zum Zeitpunkt gerade pensioniert war, antwortete Folgendes auf die Frage, ob es etwas gebe, das sie sich noch selbst für die $\mathrm{Zu}-$ kunft wünsche:

"Also, die, die - meine Freundin hat das gesagt. Die hat einen Hirnschlag gehabt, und dann hat sie gesagt: ,Weiß du, wie ich da aufgewacht bin, dann habe ich mir eine Liste genommen und habe diese letzte Löffelliste - oder so, hat sie - hat sie die genannt. Bevor sie den Löffel abgibt, möchte sie noch das und das und das machen so quasi. Habe ich mir überlegt: Möchtest du das auch? Gäbe es jetzt etwas für dich, was du noch machen möchtest? Und dann ist mir eigentlich nur so gekommen: Ich hatte ein reiches Leben, und es gibt nichts, was ich jetzt glaube, noch erleben zu müssen. Alles, was ich jetzt noch kriege, ist ein Bonbon, ein Geschenk dazu.«

Frau Witt stellt sich in ihrer Erzählung als jemand dar, der an der existenziellen Bedrohung gereift ist, der tiefgründig wurde, der die verbleibende Zeit reevaluiert. Sie sieht es im Sinne einer Selbstpositionierung als Chance beziehungsweise als Vorteil ihrer Krankheit, sich auf die ihr wichtigen Dinge im Leben zu fokussieren. Sie integriert eigene Erfahrungen vom Tod ihrer Mutter und distanziert sich von denjenigen Menschen, die beim Gedanken an den bevorstehenden Tod konkrete Ziele wie Reisen in den Blick nehmen im Sinne einer Fremdpositionierung. Frau Steinbach dagegen reflektiert den Vorgang des Erstellens einer metaphorisch genannten »letzten Löffelliste«. Sie grenzt sich davon ab, überhaupt noch eine Liste zu brauchen, weil sie auf ihr Leben voller Zufriedenheit zurückblicken kann. Während Frau Witt explizit auf die Möglichkeit »wenn ich jetzt sterbe« zu sprechen kommt, referiert Frau Steinbach implizit sowie metaphorisch auf die Überlegung ihrer Freundin, deren Aufwachen nach dem Hirnschlag und die Löffelliste - ohne den eigenen Tod oder das eigene Sterben auszusprechen. Gemeinsam ist den beiden die jeweils explizite Betonung der Bilanzierung der verbleibenden Lebenszeit als "Geschenk«, als "zusätzliche Zeit», als 
"Bonbon«. Sie drücken darin tiefe Dankbarkeit aus, die sie aus der Lebensbedrohung gewonnen haben.

Diese Dankbarkeit und Bilanzierung des Lebens sowie Reevaluierung der verbleibenden Zeit wird innerhalb der Psychotherapieforschung auch als "posttraumatic growth $"{ }^{11}$ oder posttraumatisches Wachstum beschrieben. Darunter wird verstanden, dass Menschen nach einem traumatisch erlebten Ereignis ihr Leben in einem anderen Licht sehen, ihre Prioritäten neu ordnen, eine positive Sicht auf ihre Lebensgestaltung bekommen und unter Umständen nach mehr Zufriedenheit in ihrem Leben streben. Bei onkologischen Patienten wird primär nicht davon gesprochen, dass sie im psychopathologischen Sinn traumatisiert wurden - trotzdem lässt sich das Konzept des posttraumatischen Wachstums auf die dadurch ausgelöste Konfrontation mit dem Tod übertragen. Der Moment des Innehaltens und Überdenkens ist Traumapatienten, Patienten nach einem Ereignis wie einem "Hirnschlag" (wie von der oben zitierten Frau Steinbach erwähnt), und Krebspatienten gemeinsam. ${ }^{12}$ Betrachtet man die sprachliche Verarbeitung von Traumanarrativen und die der Krebspatienten, so fällt auf, dass es sich bei der Konfrontation mit der Endlichkeit oft um schwerlich beschreibbare existenzielle Erfahrungen handelt. Die emotionalen Aspekte dieser Erfahrungen stellen die Erzählerinnen vor interaktionelle Herausforderungen. Im oben beschriebenen Forschungsprojekt wurde ein Ambivalenzmodell des Sprechens über Tod und Sterben erarbeitet, das die Funktionen des Erzählens (Ausdruck von Kontrolle, Sinnherstellung, soziale Integration und Emotionsaktualisierung) mit den sprachlichen Strategien (Metaphern, Perspektivierung, Humor, Zuhöreradressierung und Unbeschreibbarkeit) vereint. ${ }^{13}$ Dies ist ebenso vergleichbar mit dem dualen Prozessmodell der Traumaverarbeitung ${ }^{14}$, das die Ambivalenz

\footnotetext{
${ }_{11}$ Vgl. hierzu z.B. Kate Hefferon/Madeleine Grealy/Nanette Mutrie, Transforming From Cocoon to Butterfly: The Potential Role of the Body in the Process of Posttraumatic Growth, in: Journal of Humanistic Psychology 50 (2010), 224-247; Kelli N. Triplett et al., Posttraumatic growth, meaning in life, and life satisfaction in response to trauma, in: Psychological Trauma:Theory, Research, Practice, and Policy 4 (2012), 400-410.

12 Klaus Gerdes bezeichnete die Konfrontation mit der Endlichkeit bei der 2. Jahrestagung der Deutschen Arbeitsgemeinschaft für Psychoonkologie e.V. in Bad Herrenalb 1984 sehr passend als "Sturz aus der normalen Wirklichkeit«.

${ }_{13}$ Adami, Zwischen Annäherung und Distanzierung.

${ }^{14}$ Carl Eduard Scheidt/Gabriele Lucius-Hoene, Kategorisierung und narrative Bewältigung bindungsbezogener Traumaerfahrungen im Erwachsenenbindungsinterview, in: Narrative Bewältigung von Trauma und Verlust, hg. v. Carl Eduard Scheidt/ Gabriele Lucius-Hoene/Anja Stukenbrock/Elisabeth Waller, Stuttgart 2015, 26-38.
} 
zwischen Annäherung und Distanzierung an das Trauma ausdrückt, wie es beim Sprechen über Tod und Sterben erkennbar ist. Die Erzähler bewegen sich in ständiger psychischer Ambivalenz zwischen Annäherung an die sprachlich attraktive Auseinandersetzung mit dem eigenen Tod und dem Tabu, das diese sowohl gesellschaftlich als auch innerpsychisch darstellt.

\section{Der Zugang zur existenziellen Perspektive durch die Konfrontation mit dem Tod oder: Was wir von Krebspatienten vielleicht lernen können}

"Wir haben mit dem Geschichtenerzählen aufgehört, als uns die Zeit abhanden kam, innezuhalten, nachzudenken und zu staunen. Das Leben rast an uns vorbei, und nur wenige Menschen sind stark genug, das Tempo aus

eigner Kraft zu drosseln. Meist sind es unvorhergesehene Ereignisse, die uns zum Innehalten zwingen und uns Zeit schenken, uns an den $\gg$ Küchentisch des Lebens` zu setzen, unsere eigene Geschichte zu verstehen und zu erzählen, aber auch, den Geschichten anderer Menschen zu lauschen und zu erkennen, dass die Welt aus lauter solchen Geschichten besteht.» Rachel Naomi Remen, Kitchen table wisdom ${ }^{15}$

Hinter dem Erzählen über Tod und Sterben im Sinne einer narrativen Bewältigung steht die psychologische Herangehensweise zwischen Angst und Furcht zu unterscheiden: Angst ist etwas Ungreifbares, wie ein großes dunkles Etwas, das einem im Nacken sitzt, während Furcht nur vor etwas Konkretem erlebt wird. Wenn wir die undefinierte Angst aus dem Nacken hervorholen, vor uns legen und somit als konkrete Furcht betrachten, wird aus etwas unendlich großem Ungreifbaren etwas Sicht- und Begreifbares. Das hilft uns aus psychologischer Sicht, besser damit umzugehen, es in unser Leben zu integrieren. ${ }^{16}$ Hier lässt sich die Angst vor dem Tod aufgrund einer konkreten Bedrohung wie einer Krebserkrankung mit der neurotischen Angst vor Versagen, Verlust, Entwertung und Tod verbinden.

Aus der psychotherapeutischen Praxis ist bekannt, dass der $\mathrm{Zu}-$ gang zur eigenen Endlichkeit eine bedeutende Intervention sein

\footnotetext{
${ }^{15}$ Rachel Naomi Remen, Kitchen table wisdom - Geschichten, die heilen, München 2007, 18f.

${ }^{16}$ Vgl. hierzu auch: Norman Straker, Facing Cancer and the Fear of Death. A Psychoanalytic Perspective on Treatment, Lanham 2013.
} 
kann, um a.) Entscheidungen, die sonst schwer fallen, zu treffen beziehungsweise zu überdenken und b.) für das persönliche Leben bedeutsame psychische Themen zu aktualisieren. Yalom beschreibt dies in seinem Werk Existenzielle Psychotherapie ${ }^{17}$ als Grundsatz einer gelungenen Psychotherapie: die Fähigkeit, sich mit dem eigenen Tod auseinanderzusetzen, um sich dem Leben zu stellen.Yalom nennt als einen wichtigen Grundsatz in der existenziellen Psychotherapie die existenzielle Schuld:»Jemand, der es versäumt, so erfüllt wie möglich zu leben, erfährt ein tiefes, mächtiges Gefühl, das ich hier als >existenzielle Schuld bezeichne. $\|^{18}$ Bei der existenziellen Schuld geht es nicht um neurotische Schuld, sondern um die Art der Selbstaktualisierung, wie sie u.a. in der Gesprächspsychotherapie bei Rogers ${ }^{19}$ beschrieben ist. Die Selbstaktualisierung im Zusammenhang mit der eigenen Endlichkeit meint, dass genau an diesem zentralen Punkt der Angst Werte, Gefühle und Autonomiestreben eines Menschen Ausdruck finden. Genau diejenigen psychologischen Bereiche, die die eigene Handlungsmächtigkeit in den Vordergrund stellen, werden bei der Auseinandersetzung mit dem Tod herausgefordert, da der eigene Tod nicht erlebt werden kann, dafür aber antizipatorisch in verschiedensten Formen und Topoi erzählt und somit im Sinne einer narrativen Identität kontrollierbar werden kann.

Frede $^{20}$ schlägt vor, im Rahmen der Psychotherapie sich dem Tod anzunähern, indem man mit dem Tod selbst in einen Dialog eintritt. Sie beschreibt, dass Patienten danach schildern, davon profitiert zu haben, mutiger geworden seien und sich nunmehr klarer für ihre eigenen Wertvorstellungen einsetzen würden. Sie erlangten eine neue Sicht von sich selbst.

Nicht nur für Menschen, die offensichtlich eine zum Tode führende Erkrankung haben, sondern auch für Patienten mit unklarer Prognose und erst recht für zum Zeitpunkt des Gesprächs vermeintlich körperlich gesunde Menschen und deren Gesprächspartner kann es schwierig sein, über den Tod zu sprechen. Umso befreiender kann es sich jedoch anfühlen, wenn es gelingt, »dem Tod seinen Stachel zu nehmen ${ }^{21}$, indem man über ihn spricht, ihn narrativ in verschiede-

\footnotetext{
${ }^{17}$ Irvin D.Yalom, Existenzielle Psychotherapie, Bergisch Gladbach 2010.

${ }^{18}$ Ebd., 324.

${ }^{19}$ Carl R. Rogers, A theory of therapy, personality, and interpersonal relationships, as developed in the client-centered framework, in: Psychology: A Study of a Science Study I. Conceptual and Systematic, hg. v. Siegmund Koch, New York 1959, 184-256. ${ }^{20}$ Ursula Frede, »Ertragt mich, dass ich rede«. Möglichkeiten der Psychodrama-Therapie bei der Begleitung Schwerstkranker, Wiesbaden 2012.

${ }^{21}$ Peter Weyland, Psychoonkologie - das Erstgespräch und die weitere Begleitung, Stuttgart 2013, 136.
} 
nen Formen verhandelt. Die Übertragung der Konfrontation mit der eigenen Endlichkeit von schwer kranken oder sterbenden Menschen auf die von Menschen, die sich an anderen Punkten ihres Lebens die Sinnfrage stellen, findet neben psychologischer Fachliteratur immer wieder Einzug in die populärwissenschaftlichen Veröffentlichungen. So schaffte es Top five regrets of the dying von Bronnie Ware ${ }^{22}$ weltweit auf die Bestsellerlisten. Es wird unter der Beschreibung realer Schicksale eine Art "memento mori« wachgerufen mit dem Appell des "carpe diem", dem Rat, das eigene Leben so zu gestalten, dass es als ein glückliches Leben gesehen werden kann, wenn man am Lebensende zurückblickt.

$\mathrm{Zu}$ diesem Prozess der Auflösung der Unkontrollierbarkeit und der grenzenlosen Angst durch das gemeinsame Erzählen über Tod, Sterben und Endlichkeit gehören letztlich beide: die Erzählerin und die Zuhörerin. All die narrativen Prozesse finden immer im zwischenmenschlichen Raum der Begegnung statt, die auch die Dimension einer spirituellen Begegnung eröffnet. Ich sage häufig zu Patienten innerhalb eines psychotherapeutischen Prozesses: "Was die Endlichkeit betrifft, sitzen wir im genau gleichen Boot" - und drücke dabei eine zutiefst überzeugte Gleichberechtigung in der Psychotherapie aus, die beim Erzählen über Tod und Lebenssinn so klar und deutlich wird wie bei keinem anderen Thema.

»We die.That may be the meaning of life. But we do language. That may be the measure of our lives. ${ }^{23}$

\footnotetext{
- Sandra Adami arbeitet als Psychologin, Psychoonkologin und Gesprächspsychotherapeutin an einer psychosomatischen Rehabilitationsklinik sowie als Dozentin an der Universität Freiburg im Breisgau. Zu ihren Arbeitsgebieten gehören insbesondere Erzählanalysen, Psychoonkologie, humanistische Psychotherapie, Palliative Care und Burnout.
}

\footnotetext{
${ }^{22}$ Bronnie Ware, The top five regrets of the dying. A life transformed by the dearly departing, London 2012.

${ }_{23}$ Toni Morrison, Nobel Lecture (1993): http://www.nobelprize.org/nobel_prizes/ literature/laureates/1993/morrison-lecture.html (10.11.2016).
} 